\title{
Lithium-mediated protection of hippocampal cells involves enhancement of DNA-PK-dependent repair in mice
}

\author{
Eddy S. Yang, Hong Wang, Guochun Jiang, Somaira Nowsheen, \\ Allie Fu, Dennis E. Hallahan, and Fen Xia
}

Department of Radiation Oncology, Vanderbilt-Ingram Cancer Center, Vanderbilt University School of Medicine, Nashville, Tennessee, USA.

\begin{abstract}
Long-term neurological deficiencies resulting from hippocampal cytotoxicity induced by cranial irradiation (IR) present a challenge in the treatment of primary and metastatic brain cancers, especially in children. Previously, we showed that lithium protected hippocampal neurons from IR-induced apoptosis and improved neurocognitive function in treated mice. Here, we demonstrate accelerated repair of IR-induced chromosomal double-strand breaks (DSBs) in lithium-treated neurons. Lithium treatment not only increased IR-induced DNA-dependent protein kinase (DNA-PK) threonine 2609 foci, a surrogate marker for activated nonhomologous end-joining (NHEJ) repair, but also enhanced double-strand DNA end-rejoining activity in hippocampal neurons. The increased NHEJ repair coincided with reduced numbers of IR-induced $\gamma-\mathrm{H} 2 \mathrm{AX}$ foci, well-characterized in situ markers of DSBs. These findings were confirmed in vivo in irradiated mice. Consistent with a role of NHEJ repair in lithium-mediated neuroprotection, attenuation of IR-induced apoptosis of hippocampal neurons by lithium was dramatically abrogated when DNA-PK function was abolished genetically in SCID mice or inhibited biochemically by the DNA-PK inhibitor IC86621. Importantly, none of these findings were evident in glioma cancer cells. These results support our hypothesis that lithium protects hippocampal neurons by promoting the NHEJ repair-mediated DNA repair pathway and warrant future investigation of lithium-mediated neuroprotection during cranial IR, especially in the pediatric population.
\end{abstract}

\section{Introduction}

Cranial irradiation (IR) as part of standard treatment of primary and metastatic brain tumors often results in long-term neurological sequelae, especially in young children (1-4). Intellectual impairment, reduction in performance IQ, memory loss, and dementia have been reported after exposure of the brain to radiation. Evidence suggests that the cognitive decline seen is due to IR-induced damage to the hippocampus, a critical area of the brain responsible for learning and memory (5-7). Consistent with these findings, radiation to the hippocampus is associated with more pronounced cognitive deficits compared with radiation to other areas of the brain (8).

The exact mechanisms of IR-induced brain injury remain to be elucidated. In general, radiation results in different forms of DNA damage, with the most critical lesion being chromosomal doublestrand breaks (DSBs) (9). Even a single unrepaired DSB can be lethal. These DSBs can be measured by the neutral comet assay $(10,11)$. They are also evident in situ and can be quantified by the number of $\gamma-\mathrm{H} 2 \mathrm{AX}$ foci formed in the nucleus $(12,13)$. Repair of DSBs occurs via 2 principal pathways, homologous recombination repair (HR) and nonhomologous end-joining (NHEJ) repair. HR is a high-fidelity, error-free process that requires an intact homologous DNA sequence as the template. It is critical in the repair of

Authorship note: Eddy S. Yang and Hong Wang contributed equally to this work. Conflict of interest: The authors have declared that no conflict of interest exists. Nonstandard abbreviations used: DNA-PK, DNA-dependent protein kinase; DNA$\mathrm{PK}_{\mathrm{cs}}$, DNA-PK catalytic subunit; DSB, double-strand break; GSK-3, glycogen synthase kinase-3; HR, homologous recombination repair; IR, irradiation; NHEJ, nonhomologous end-joining.

Citation for this article: J. Clin. Invest. 119:1124-1135 (2009). doi:10.1172/JCI34051. lesions resulting from replicative stress. Multiple proteins, including the $\operatorname{Rad} 51 / \operatorname{Rad} 52$ recombinases, BRCA1, and BRCA2, are involved in this intricate process.

In contrast, NHEJ repair is a highly efficient but error-prone process that often results in mutations in the repaired DNA. It is the predominant repair mechanism for DSBs resulting from IR (14, $15)$. The NHEJ repair process is dependent on the DNA-dependent protein kinase (DNA-PK) catalytic subunit (DNA-PK $\mathrm{C}_{\mathrm{cs}}$ ), the $\mathrm{Ku} 70 / \mathrm{Ku} 80$ heterodimer, and the XRCC4-ligase IV complex and ultimately rejoins the ends of DSBs with little or no homology. In response to IR, DNA-PK $\mathrm{PK}_{\mathrm{cs}}$ is autophosphorylated at threonine 2609 (T2609) in vivo, which is required for the functional activation of the NHEJ repair pathway (16). Phosphorylated DNA-PK has also been shown to colocalize with DNA damage response proteins such as $\gamma-\mathrm{H} 2 \mathrm{AX}$ at sites of DSBs, and T2609 foci formation is a well-characterized surrogate marker for activated NHEJ repair $(15,16)$. Consistent with the role of NHEJ repair in the repair of IR-induced DSBs, cells deficient in any NHEJ repair protein have been shown to be hypersensitive to IR-mediated cytotoxicity (13-16). Interestingly, genetic knockout of NHEJ repair proteins in mice has also been shown to increase p53-dependent cell death of neurons and results in deficits in neurogenesis (17-20).

In an effort to decrease neurotoxicity and improve patient quality of life, pharmacologic agents that exhibit neuroprotective effects are being rigorously investigated. One such potential compound is lithium, a drug widely used in the treatment of bipolar mood disorder (21). Evidence suggests that lithium protects the brain against a variety of insults, such as stroke and oxidative stress $(6,7,21-25)$. However, the mechanisms of the neuroprotection by lithium are not well defined. Several studies have reported that lithium activates the prosurvival PI3K/Akt signaling pathway, 
leading to inhibition of the glycogen synthase kinase-3 (GSK-3) pathway $(6,26,27)$. Recently, we have shown that lithium prophylaxis improves cognitive performance in mice exposed to cranial IR and protects irradiated hippocampal neurons from apoptosis (6).

Chromosomal DSBs are the most critical lesions for IR-induced cell death. We therefore investigated whether lithium-mediated regulation of the DNA damage/repair pathways can be a potential mechanism of neuroprotection from IR-induced apoptosis. Using the neutral comet assay, a gel-based method to detect and assess cellular chromosomal breaks $(11,28)$, and $\gamma$-H2AX foci, a wellcharacterized in situ marker of DNA DSBs, we demonstrated that lithium did not affect the initial number of cells exhibiting DSBs after IR, but decreased the percentage of mouse hippocampal neurons with persistent DSBs, suggesting a role of DSB repair in lithium-mediated neuroprotection. Further dissection of the mechanisms responsible for the potential enhanced repair of IR-induced DSBs revealed that lithium significantly increased NHEJ repair in hippocampal cells, as evidenced not only by evaluation of DNA-PK T2609 foci, but also by direct assays of cellular capacity for end-rejoining of DSB. These neuroprotective effects by lithium were validated in vivo by immunohistological examination of hippocampal tissue from irradiated C57BL/6J mice. In support of a role for NHEJ repair, lithium-mediated protection of IR-induced apoptosis was diminished in DNA-PK-deficient hippocampal neurons in SCID mice and was attenuated in normal hippocampal cells by the DNA-PK inhibitor 1-(2-hydroxy-4morpholin-4-yl-phenyl) ethanone (designated IC86621; ref. 29). More importantly, these lithium-mediated effects were not evident in glioma cancer cells. Our results revealed a mechanism, which we believe to be novel, of lithium-mediated neuroprotection through enhancing NHEJ repair-mediated DNA DSB repair in irradiated noncancerous neuronal cells.

\section{Results}

Lithium enhances repair of DNA DSBs after IR. The critical cellular lesions in relation to cytotoxic effects generated by IR are DNA DSBs. Because lithium has been shown to protect irradiated neurons from apoptosis (6), we investigated whether lithium-mediated neuroprotection involves regulation of the DNA damage/repair pathways. We first assessed the repair kinetics of IR-induced DSBs using the neutral comet assay $(10,11)$ in irradiated hippocampal neuronal cells with or without lithium prophylaxis at various time points after IR. Similar levels of IR-induced DSBs, as evidenced by percentage of cells exhibiting a comet tail (Figure 1, A and B) as well as the calculated mean tail moment (Figure 1C), were observed in both lithium- and vehicle-treated HT-22 hippocampal cells at 15 minutes after IR. However, at 30 minutes after IR, accelerated repair of IR-induced DSBs was observed in lithium-treated neuronal cells compared with cells not exposed to lithium. The difference of persistent DSBs continued at 8 hours after IR. By 24 hours after IR, DSBs returned to basal levels in all groups (Figure $1, B$ and C). These findings suggest that lithium affects the DNA DSB repair pathways, but not the initial DSB formation after IR.

To further assess the potential role of enhanced repair of IRinduced DSBs by lithium, we also examined $\gamma$-H2AX foci, a commonly used in situ marker of DNA DSBs, in irradiated neuronal cells with and without lithium prophylaxis. Lithium prophylaxis for 7 days did not affect the initial percentage of cells with elevated IR-induced $\gamma$-H2AX foci, but dramatically decreased the percentage of cells exhibiting persistent $\gamma-\mathrm{H} 2 \mathrm{AX}$ foci in mouse
HT-22 hippocampal neurons (Figure 2, A and B). The maximal reduction in the number of cells with persistent DSBs was seen 1 hour after IR (2.4-fold), while the levels of $\gamma$-H2AX foci returned to basal levels by 24 hours after IR (Figure 2A). This effect was similarly observed in mouse primary neurons (maximal 3.5-fold at 1 hour after IR; Figure 2C). Additionally, lithium did not affect the initial mean number of IR-induced $\gamma-\mathrm{H} 2 \mathrm{AX}$ foci per cell, but attenuated the accumulation of persistent numbers of $\gamma$-H2AX foci per cell (maximal 1.7-fold at 1 hour after IR; Figure 2D). These data again support the notion that lithium-mediated neuroprotection of hippocampal neurons involves enhanced repair of IR-induced DSBs.

Lithium enhances NHEJ repair, but not $H R$. To determine the mechanisms of lithium-mediated induction of DSB repair in neuronal cells, we examined the in vivo functional activities of HR and NHEJ repair, the 2 major DSB repair pathways, in irradiated neurons with and without lithium prophylaxis. We first assessed lithium-mediated effects on NHEJ repair in irradiated neurons. NHEJ repair is the predominant repair mechanism for DSBs resulting from IR (12-14). Radiation has been shown to activate NHEJ repair by inducing autophosphorylation of DNA-PK ${ }_{\mathrm{cs}}$ at T2609 (12-14). It has also been well established that phosphorylated DNA-PK nuclear foci detected by the phospho-T2609-specific antibody (referred to herein as T2609 foci) can be used as an in vivo functional marker of NHEJ repair activity (16). We thus examined T2609 foci to evaluate NHEJ repair in irradiated neurons. Lithium enhanced the percentage of cells with IR-induced T2609 foci in mouse HT-22 neuronal cells (Figure 3, A and B) and in mouse primary hippocampal neurons (Figure 3, C and D). The significant increase in number of cells with elevated T2609 foci was seen as early as 0.5 hours and persisted 4.0 hours after IR. There was a maximal 1.6-fold enhancement in the percentage of cells exhibiting elevated levels of IR-induced T2609 foci by lithium at 1.0 hour after IR. The mean number of foci per cell was similarly enhanced by lithium in primary neurons (maximal 1.5 -fold at 0.5 hours after IR; Figure 3D). Interestingly, these findings were coincident with the maximal decrease in the number of cells with persistent IR-induced $\gamma$-H2AX foci by lithium, which suggests that lithium may attenuate the persistence of IR-induced DSBs via induction of DNA-PK dependent NHEJ repair.

To confirm a role of NHEJ repair in lithium-mediated increase in repair of IR-induced DSBs, a direct biochemical in vivo assay measuring intramolecular NHEJ repair was performed using a well-established plasmid-based episomal DSB end-rejoining assay (30). In these experiments, the plasmid substrate pEGFP-N1 was cleaved using the EcoRI restriction endonuclease in vitro between the promoter and the GFP reporter gene, thereby preventing expression of the reporter in vivo (Figure 4A). This linearized plasmid was cotransfected with an internal control circularized plasmid, pdsRed. Intracellular recircularization of the linearized DNA through NHEJ repair-mediated end-rejoining allows the expression of GFP, which was then assayed by 2 -color flow cytometric analysis. The rejoining levels in lithium-treated hippocampal cells were significantly enhanced by more than 2 -fold compared with control cells (Figure 4B). These findings provide direct evidence that lithium enhances NHEJ repair-mediated repair of DSBs.

We next analyzed HR, the other DSB repair pathway, in irradiated neurons by investigating the effects of lithium on IR-induced Rad51 foci, an in vivo functional marker of HR (31). In contrast to the effects on NHEJ repair, pretreatment with lithium slightly 
A
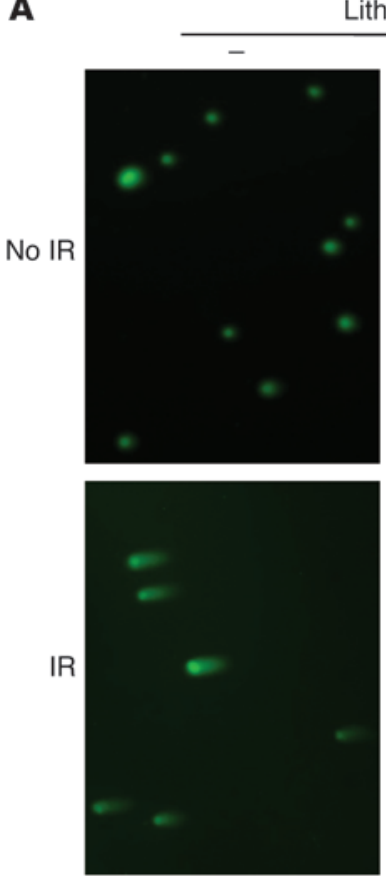

Lithium
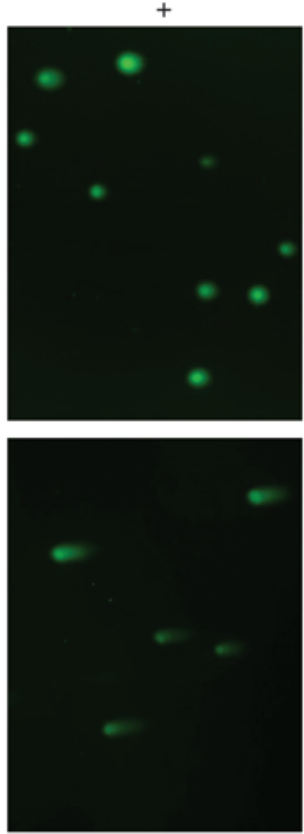

B
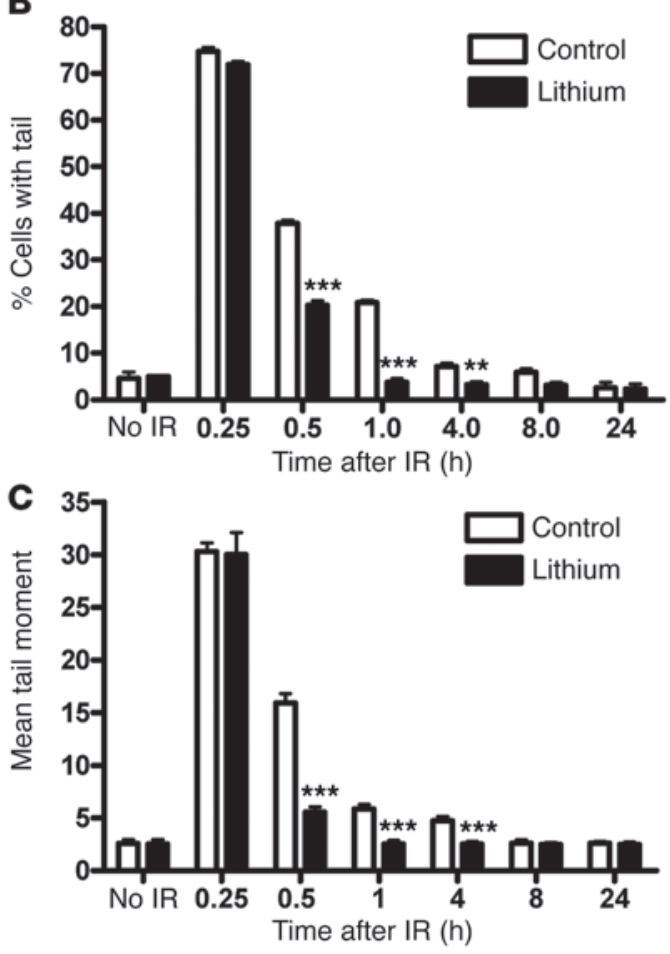

Figure 1

Lithium enhances repair of IR-induced DSBs in hippocampal neurons. HT-22 neurons were treated with 3 mM lithium or vehicle for 7 days. Following the treatment period, cells were exposed to $3 \mathrm{~Gy}$ IR and incubated for the indicated times. Cells were then prepared and subjected to neutral comet assay as described in Methods. At least 75 comet images were analyzed. (A) Representative control and irradiated cells with or without lithium prophylaxis with and without tails. Original magnification, $\times 400$. (B) Mean percent of cells with comet tail, indicative of DSBs, after IR at various time points. (C) Mean tail moment in control and irradiated cells with and without lithium prophylaxis at various time points after IR. Error bars represent SEM. ${ }^{\star \star} P<0.01,{ }^{\star \star \star} P<0.001$ versus control.

decreased the percentage of cells with IR-induced Rad51 foci in HT-22 cells (Figure 5A) and in mouse primary hippocampal neurons (Figure 5B). Similar to the kinetics of lithium-mediated effects on NHEJ repair, the greatest reduction was observed at 0.5 hours after IR. These results are consistent with those of previous studies that have shown HR to be a minor component of IR-induced DNA repair (reviewed in ref. 14). Alternatively, decreased HR activity may be secondary to upregulation of DNA-PK-dependent NHEJ repair capacity and enhanced DSB repair efficiency (32).

Lithium upregulates NHEJ repair of IR-induced DSBs in vivo. To validate our findings of lithium-mediated induction of NHEJ repair in vivo, we first explored IR-induced DSBs in irradiated C57BL/6J mice with or without 7 days of lithium prophylaxis. Histological analysis of hippocampal neurons from the brains of these mice (Figure 6A) revealed that lithium decreased the percentage of hippocampal neurons with persistent $\gamma-\mathrm{H} 2 \mathrm{AX}$ foci, with a maximal 5 -fold decrease at 1 hour after IR (Figure 6B). Interestingly, similar to hippocampal cells in culture, there was no difference in the percentage of cells with residual IR-induced $\gamma$-H2AX foci with or without lithium treatment at the early time points. Again, these kinetics suggest that lithium may not be markedly affecting the generation of DSBs during IR, but rather is enhancing DSB repair.

We next assessed DSB repair in irradiated mice with or without lithium prophylaxis by examining the functional activities of $\mathrm{HR}$ and NHEJ repair. Similar to irradiated HT-22 cells (Figure 3A) and mouse primary neurons (Figure $3 \mathrm{~B}$ ), lithium increased the percentage of cells with elevated DNA-PK T2609 foci - which are in vivo markers of NHEJ repair activity $(15,16)$ - in histologic sections from mouse hippocampal brain tissue (Figure 6C). This significant increase occurred as early as 0.5 hours after IR and persisted 4.0 hours after IR. There was a maximal 1.6 fold-enhancement of cells with elevated IR-induced T2609 foci by lithium, which occurred 1 hour after IR.

To examine lithium-mediated effects on HR in irradiated mice, we investigated IR-induced Rad51 foci, an in vivo functional marker of HR (31). As shown in HT-22 cells (Figure 5A) and mouse primary hippocampal neurons (Figure 5B), lithium also decreased the percentage of cells with Rad51 foci in hippocampal sections from irradiated mouse brains (Figure 6D). The greatest reduction, 1.25 -fold, was observed at 0.5 hours after IR. Taken together, our data strongly suggest a role of NHEJ repair, but not HR-mediated repair, of IR-induced DSBs in lithium-mediated protection of irradiated neurons.

Lithium-mediated neuroprotection requires DNA-PK-dependent NHEJ repair. To further substantiate the role of DNA-PK-dependent NHEJ repair in lithium-mediated effects on IR-induced DSBs, we next investigated the effects of the DNA-PK inhibitor IC86621 on lithium-mediated protection of neurons from IR-induced apoptosis. These studies were conducted in HT-22 cells as a representative model, since they appeared to behave similarly to mouse primary neurons and mouse hippocampal brain tissue in our previous experiments. Apoptosis of irradiated HT-22 cells was attenuated 
A

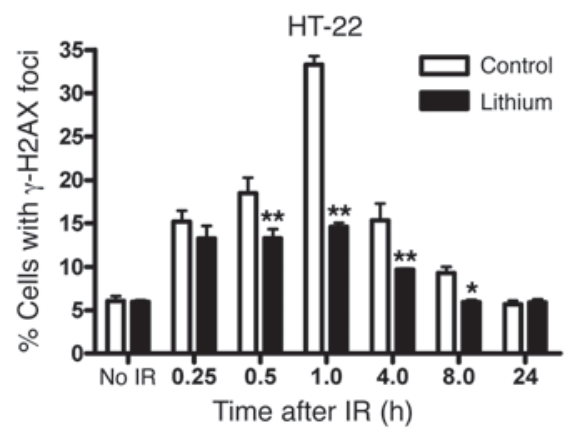

B
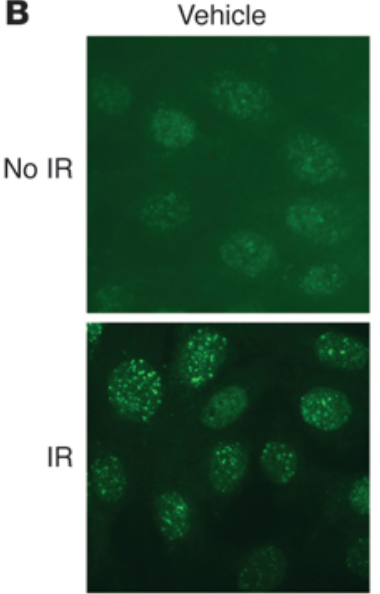

Lithium

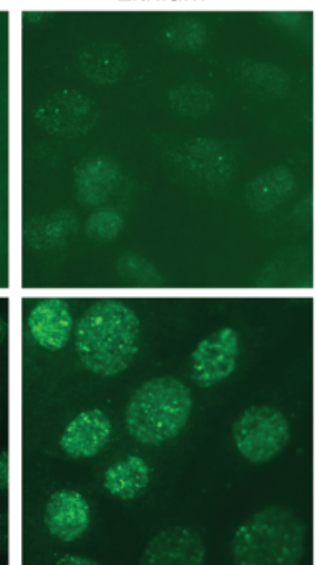

C

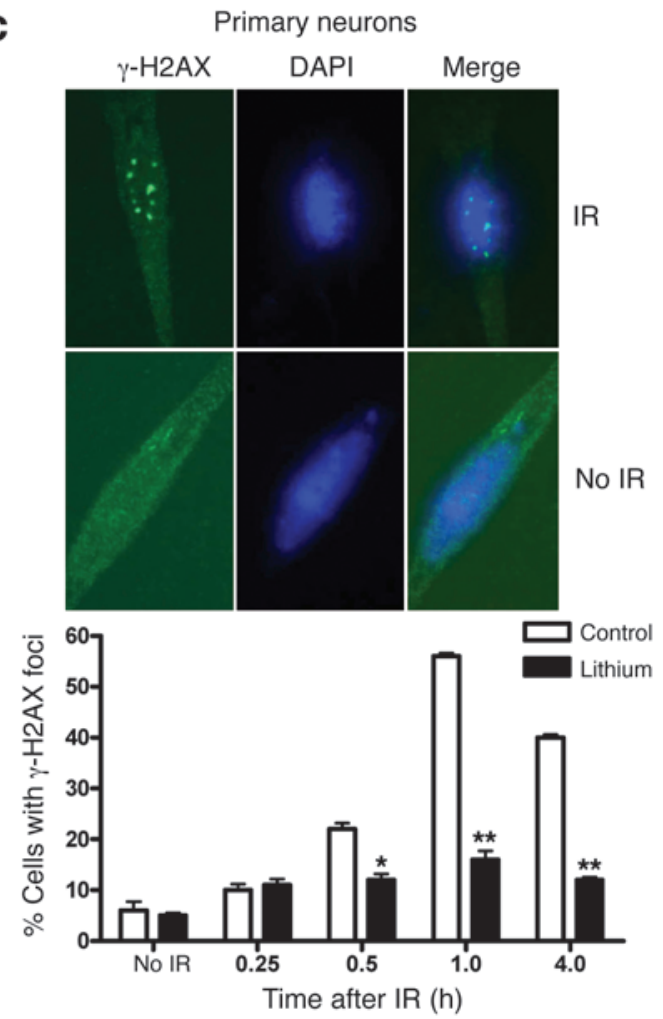

D

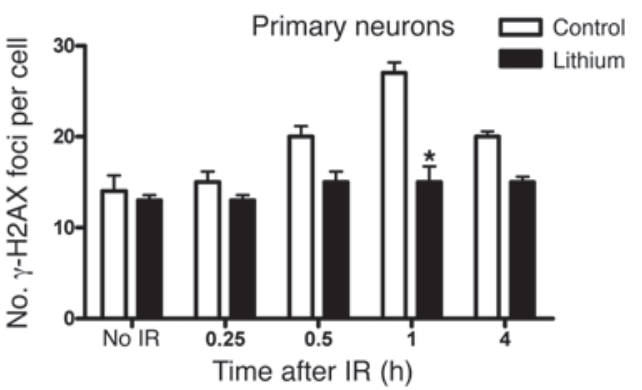

Figure 2

Lithium decreases the levels of persistent IR-induced $\gamma-\mathrm{H} 2 \mathrm{AX}$ foci. Cells were treated with $3 \mathrm{mM}$ lithium for 7 days. Following the treatment period, cells were exposed to 3 Gy. After the indicated times, cells were processed for immunofluorescence staining for $\gamma-\mathrm{H} 2 \mathrm{AX}$. Data (mean $\pm \mathrm{SEM}$ from at least 3 independent experiments) show the percentage of cells containing greater than 10 foci or the number of foci per cell, as indicated. (A) Percentage of cells with $\gamma-\mathrm{H} 2 \mathrm{AX}$ foci in HT-22 mouse hippocampal neurons. (B) Representative foci staining in HT-22 cells. Original magnification, $\times 400$. (C) The upper panel shows representative $\gamma-\mathrm{H} 2 \mathrm{AX}$ foci immunofluorescence staining in irradiated and unirradiated mouse primary neurons. The lower panel shows the percentage of cells with $\gamma-\mathrm{H} 2 \mathrm{AX}$ foci in mouse primary neurons. Original magnification, $\times 400$. (D) Number of IR-induced $\gamma-\mathrm{H} 2 \mathrm{AX}$ foci per cell, which was decreased by lithium treatment. ${ }^{\star} P<0.05,{ }^{\star \star} P<0.01$ versus control.

by lithium (2.05-fold at 24 hours after IR, 2.30-fold at 48 hours after IR; Figure 7, A and B), similar to previous reports (6). The addition of the DNA-PK inhibitor IC86621 abrogated lithiummediated neuroprotection (1.31-fold at 24 hours after IR, 1.14-fold at 48 hours after IR; Figure 7, A and B). The DNA-PK inhibitors alone sensitized cells to IR (data not shown), as previously reported (33-35). In the absence of IR, neuronal cells elicited a slight apoptotic response to the DNA-PK inhibitors (data not shown).

Our data thus far strongly supported a role of NHEJ repair in lithium-mediated protection of neuronal cells from IR-induced apoptosis. To validate this role, we hypothesized that neurons from mice genetically deficient in NHEJ repair are not protected from IR-induced apoptosis by lithium. We assessed lithium-medi- ated neuroprotection from IR-induced apoptosis in SCID mice, which are NHEJ repair deficient as a result of genetic mutation of DNA-PK $\mathrm{Cs}_{\mathrm{cs}}$, and found them to be radiosensitive (Figure 7, C and D), as previously reported (36). Irradiated wild-type mice exhibited greater than 2 -fold protection of hippocampal neurons from IR-induced apoptosis with lithium (Figure 7, C and D). In contrast, but in accordance with a role of NHEJ repair in lithium-mediated protection of neurons from IR-induced apoptosis, neurons from the NHEJ repair-deficient SCID mice treated with lithium exhibited levels of IR-induced apoptosis similar to those of vehicle-treated irradiated SCID mice ( 6 hours, 83 versus 92 apoptotic cells; 12 hours, 204 versus 212 apoptotic cells; $P>0.05$ for both comparisons; Figure 7, C and D). Taken together, these 
A

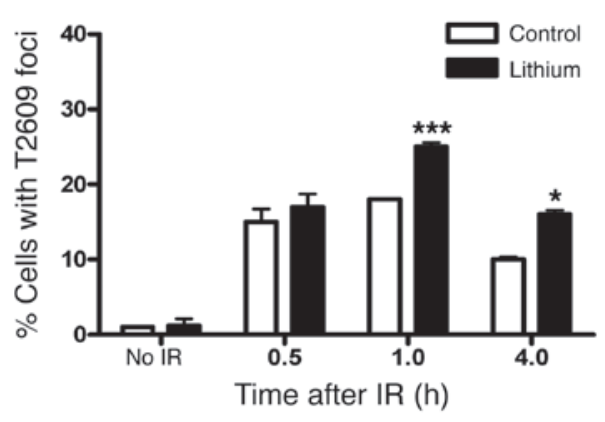

B
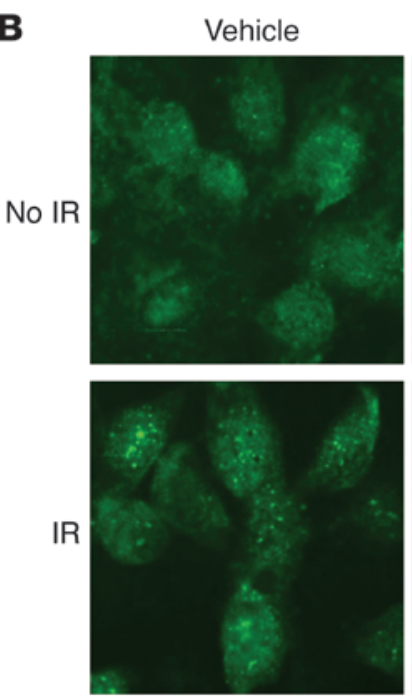

Lithium
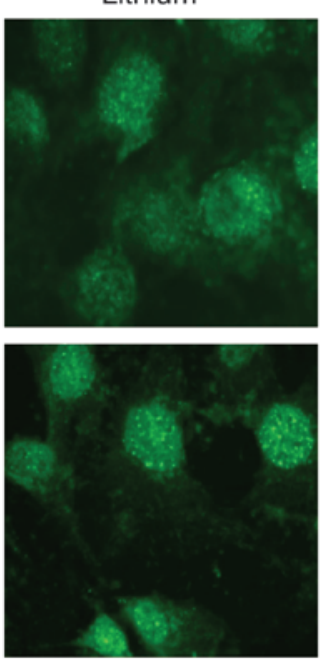

Figure 3

C
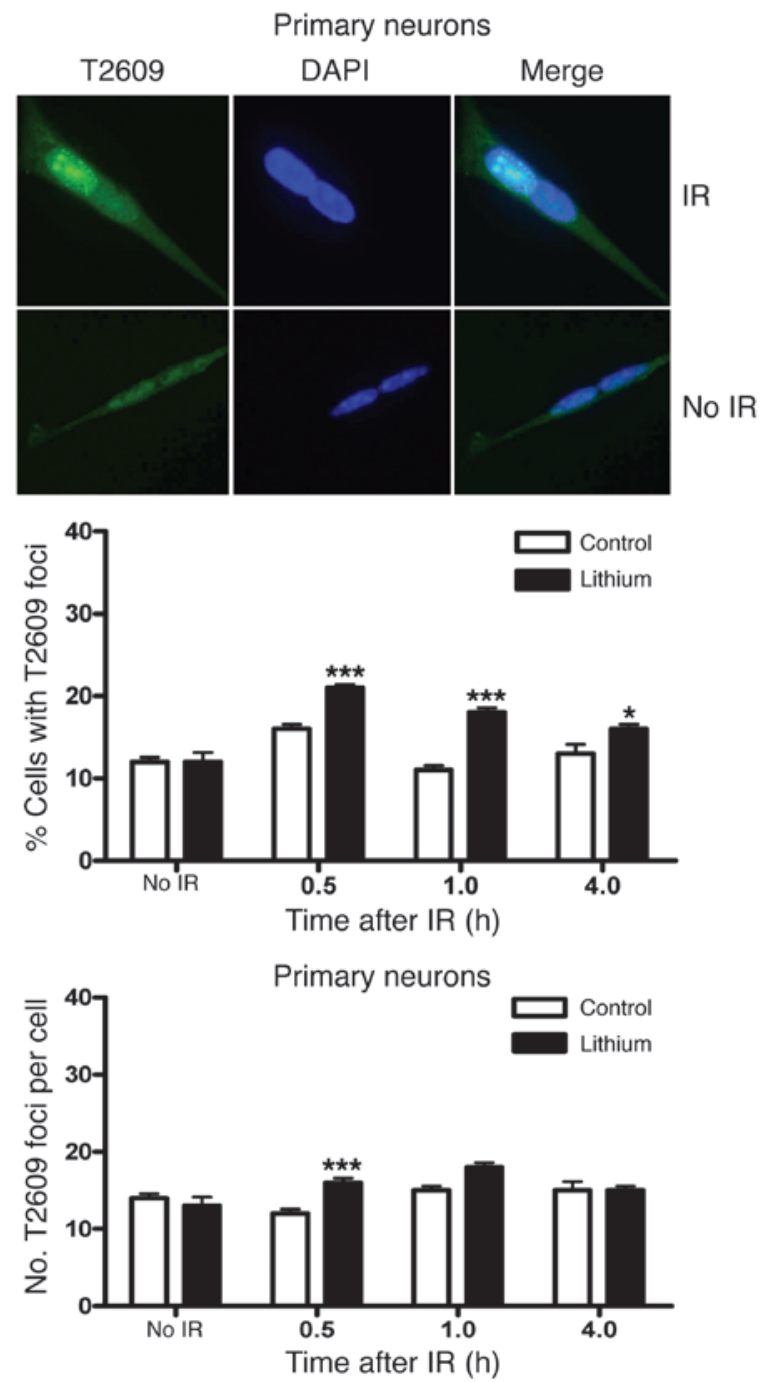

Lithium enhances DNA-PK T2609 foci. Cells were treated with $3 \mathrm{mM}$ lithium for 7 days. Following the treatment period, cells were exposed to 3 Gy. At the indicated times, cells were processed for immunofluorescence staining for DNA-PK T2609 foci. Data (mean \pm SEM from at least 3 independent experiments) show the percentage of cells containing greater than 10 foci or the number of foci per cell, as indicated. (A) Percentage of cells with elevated DNA-PK T2609 foci in HT-22 mouse hippocampal neurons. (B) Representative foci staining in HT-22 cells. Original magnification, $\times 400$. (C) The upper panel shows representative DNA-PK T2609 foci immunofluorescence staining in irradiated and unirradiated mouse primary neurons. The lower panel shows the percentage of cells with elevated DNA-PK T2609 foci in mouse primary neurons. Original magnification, $\times 400$. (D) Number of IR-induced T2609 foci per cell, which was increased by lithium treatment. ${ }^{*} P<0.05,{ }^{\star \star \star} P<0.001$ versus control.

data firmly support the notion that lithium-mediated neuroprotection from IR-induced apoptosis results at least in part from enhanced repair of IR-induced DSBs through the DNA-PKdependent NHEJ repair pathway.

Lithium-mediated neuroprotection does not occur in glioma tumor cells. We next studied whether the enhancement effect of lithium on NHEJ repair function is specific to normal neuronal cells and not to brain tumor cells. We have previously shown that radioprotection by lithium does not occur in the mouse glioma cell line GL261 (6). GL261 cells express a mutated $p 53$ gene, with a homozygous point mutation at codon 153 of exon 5 (37). These cells are rather radiosensitive in vitro, as less than 2 Gy IR can achieve $50 \%$ cell mortality (37). However, in vivo, a much higher dose of local tumor IR did not cure any animals bearing these cancer cells (37). As shown in Figure 8A, lithium did not affect the per- centage of GL261 cells with elevated IR-induced $\gamma$-H2AX foci at all time points analyzed. Consistent with a lack of radioprotection, the enhancement of NHEJ repair by lithium in neuronal cells was not evident in GL261 cells (Figure 8B), nor was a significant effect of lithium on Rad51 observed (Figure 8C). The DNA-PK inhibitors and lithium did not affect IR-induced apoptosis of these cells (Figure 8, D and E), although the addition of DNA-PK inhibitors alone still radiosensitized GL261 cells (data not shown). Similarly, lithium-mediated neuroprotection and reduction of IR-induced $\gamma-\mathrm{H} 2 \mathrm{AX}$ foci was not observed in the D54 human glioma cell line (data not shown and ref. 6). This differential effect of lithium in the glioma cancer cells versus mouse hippocampal neuronal cells further substantiated the potential clinical utility of lithium and may provide a means to improve the therapeutic index of cranial IR. 

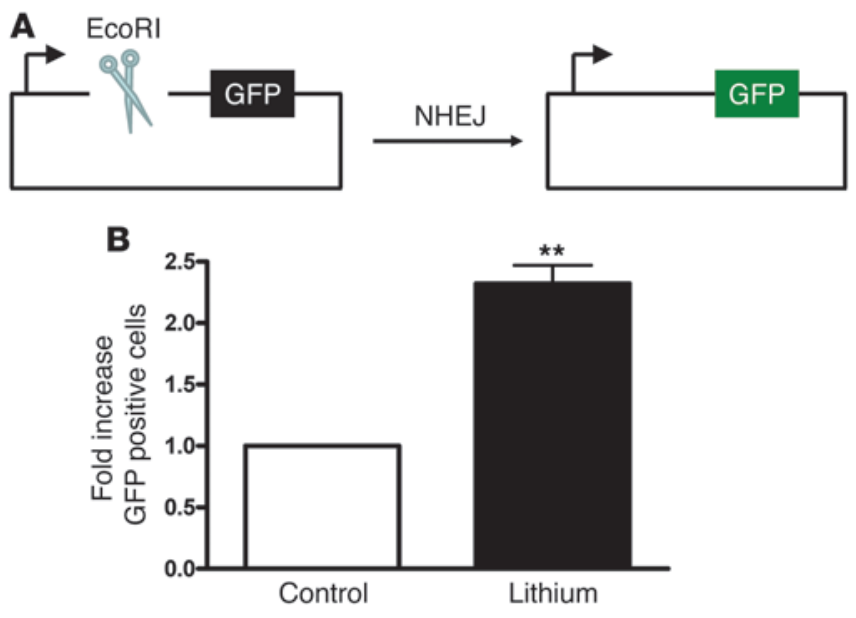

Figure 4

Lithium enhances NHEJ repair in HT-22 hippocampal neurons. (A) Schematic of the NHEJ repair assay. (B) Rejoining capacity of HT-22 neurons with or without lithium. The plasmid substrate pEGFP-N1 was linearized with the EcoRI endonuclease, which cleaves between the promoter and the GFP gene and prevents GFP expression. HT-22 cells were cotransfected with this linearized plasmid as well as the circular pdsRed as an internal control for transfection efficiency. At 48 hours after transfection, cells were harvested for 2-color flow cytometric analysis. Data (mean \pm SEM from 3 independent experiments) show the ratio of GFP expression to pdsRed expression as a function of vehicle-treated cells. ${ }^{* *} P<0.01$ versus control.

\section{Discussion}

In this report, we demonstrated that the DNA NHEJ repair pathway is a mechanism, which we believe to be novel, underlying lithium-mediated protection of normal mouse hippocampal neurons from IR-induced apoptosis. We demonstrated that IR-induced chromosomal breaks were repaired with greater efficiency in lithium-treated neuronal cells, as observed by the neutral comet assay. Furthermore, lithium enhanced DNA-PK-dependent NHEJ repair activity, which was evident not only by the increased number of cells with elevated T2609 foci after IR but also by the enhanced double-stranded DNA end-joining capacity by lithium. The increase in DNA-PK-dependent NHEJ repair activity was associated with a subsequent decrease in the percentage of cells with persistent IR-induced $\gamma$-H2AX foci, which are commonly used in situ markers of DNA DSBs. The addition of the DNA-PK inhibitor IC86621 attenuated lithium-mediated protection of hippocampal cells from IR-induced apoptosis. Furthermore, this protection was abolished in NHEJ repair-deficient SCID mice. Importantly, all of these findings were not evident in the mouse glioma cancer cell line GL261. Our present findings provide direct biochemical and genetic evidence supporting the notion that lithium protects irradiated noncancerous neuronal cells from apoptosis, in part by enhancing DNA-PK-dependent NHEJ repair.

Interestingly, although our results from the 2 DSB assays both suggest an effect by lithium on repair but not induction of DSBs after IR, the kinetics of IR-induced DNA damage and/or repair as assessed by the neutral comet assay and $\gamma$-H2AX foci were slightly different: the maximal comet tail moment was detected 15 minutes after IR via the neutral comet assay versus the delayed $\gamma$-H2AX foci peak at 1 hour after IR. This observation is consistent with several reports suggesting that while the neutral comet assay allows for the direct measurement of IR-induced DSBs, persistent or low level (<50 strand breaks) DNA damage can go undetected, especially at lower radiation doses $(10,11,38)$. Additionally, the formation of $\gamma$-H2AX foci typically requires the recruitment of DNA damage response proteins by the DSB, which may delay foci formation. Alternatively, there is the potential possibility that lithium could affect H2AX phosphorylation or subcellular localization. However, our biochemical and genetic data (Figures 4 and 8) support the notion that lithium enhances repair of IR-induced DNA damage through enhancing NHEJ repair.

The mechanisms of lithium-mediated neuroprotection have been previously attributed to activation of the prosurvival PI3K/ Akt pathway and inhibition of the GSK-3 enzyme (6, 26, 27, 39). In response to DNA damage, Akt is ultimately activated as part of the cellular survival response (40-43). This activation has been shown to require DNA-PK, which is an efficient kinase that can phosphorylate Akt at serine 473 both in vitro and in vivo after DNA damage (42-46). Regulation of these pathways in turn decreases the levels of the proapoptotic proteins p53 and Bax while enhancing expression of the antiapoptotic protein Bcl-2 (6, 22, 24, 39). Several reports have also demonstrated stabilization of the p53dependent cell cycle checkpoint protein p21 by activation of Akt or suppression of GSK-3 (47-49). Furthermore, recent studies have revealed a potential dependence of IR-induced $\mathrm{p} 53$ response on activation of DNA-PK and Akt and subsequent inhibition of GSK-3 and mdm2 $(42,50)$. As lithium affects the Akt and GSK-3 pathways, our present findings could potentially link lithium and inhibition of IR-induced apoptosis through regulation of DNA repair, in particular DNA-PK and NHEJ repair.

In addition to effects on cell survival/apoptotic pathways, lithium has previously been reported to alter cell cycle distribution in treated cells, in particular at the $\mathrm{G}_{2} / \mathrm{M}$ checkpoints (51-53). This is thought to be secondary to lithium-mediated activation of checkpoint kinase 1 (Chk1), a critical enzyme in DNA damage-induced $\mathrm{G}_{2} / \mathrm{M}$ arrest. Interestingly, we did not observe any changes in cell cycle distribution in the HT-22 hippocampal neurons at the dose and time frames used in this study (Supplemental Figure 1; available online with this article; doi:10.1172/JCI34051DS1). This may be a neuron-specific effect.

Our findings suggest a role of the DNA-PK-dependent NHEJ repair pathway in lithium-mediated neuroprotection. Consistent with a role of DNA-PK in this effect, the DNA-PK inhibitor IC86621 attenuated lithium-mediated protection of hippocampal neurons from IR-induced apoptosis. DNA-PK inhibitors are wellestablished radiation sensitizers (33-35). However, given our data suggesting a role of DNA-PK in lithium-mediated neuroprotection, the use of these inhibitors should be approached cautiously, as these compounds may decrease the therapeutic index as a result of sensitization of normal cells.

The use of lithium as a neuroprotector against brain injury has been proposed for other insults to the brain in addition to IR. Evidence suggests that lithium can protect the brain in stroke and oxidative stress, and it has been shown to reduce brain damage in animal models of neurodegenerative diseases and stroke $(6,7$, 21-25, 54). The doses of lithium used in our study are comparable to those in previous reports. Clinically, however, lithium has a relatively low therapeutic index, requiring careful blood level monitoring in light of its well-known toxicities (55), both acute (including gastrointestinal discomforts such as nausea, diarrhea, vomiting, and stomach pain; muscular weakness; thirst and frequent urina- 


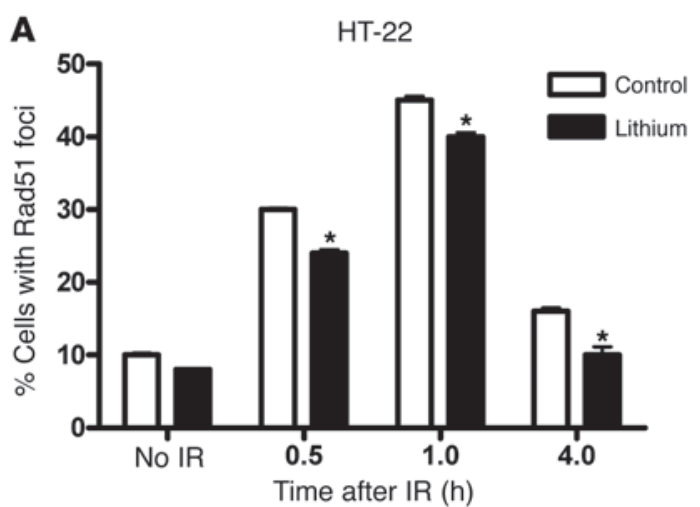

B
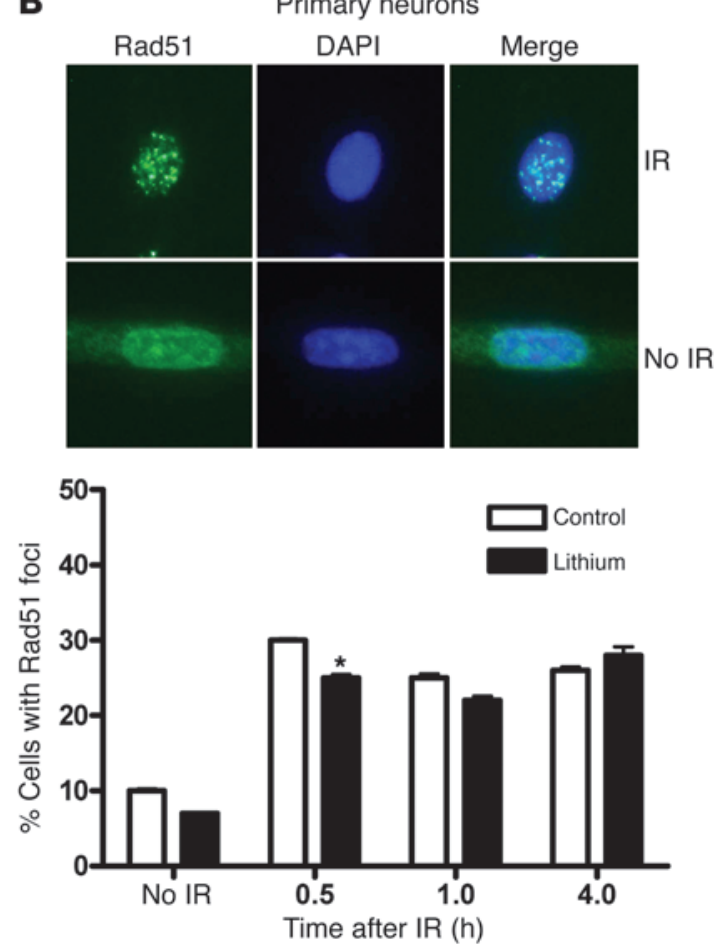

tion; feelings of being dazed, sleepy, and tired; and hand tremor) and subacute (hand tremor; constant thirst; and abundant urine excretion). In addition, the therapeutic action of lithium is delayed, requiring 5-7 days prophylaxis prior to the initiation of IR therapy to reach steady-state concentrations (56). We are currently conducting a phase I study to evaluate the safety and toxicity of lithium as a neuroprotective agent during cranial radiotherapy.

Lithium-mediated neuroprotection, however, did not occur in mouse glioma cancer cells (Figure 8 and ref. 6). This differential effect of lithium in glioma cancer cells versus hippocampal neuronal cells provides the potential to improve the therapeutic index of cranial IR. Glioma cells are notoriously resistant to radiation and chemotherapy. As further evidence for the resistance of glioma cells to IR, we did not see the number of GL261 mouse glioma cells or D54 human glioma cells with IR-induced $\gamma-\mathrm{H} 2 \mathrm{AX}$ foci demonstrate the vast increase seen in normal hippocampal neurons (maximum $8 \%$ in glioma cells versus maximum 30\%-50\% in neurons; Figure 2 and Figure 8A). This low level of cells with persistent foci was associated with an efficient induction of DNA-PK in glioma cells by IR (maxi-

\section{Figure 5}

Lithium moderately decreases the percentage of cells with elevated Rad51 foci in mouse HT-22 hippocampal and primary neurons. HT-22 mouse hippocampal neurons (A) and mouse primary neurons (B) were treated with $3 \mathrm{mM}$ lithium for 7 days. Following the treatment period, cells were exposed to $3 \mathrm{~Gy}$. At the indicated times, cells were processed for immunofluorescence staining for Rad51. Data (mean \pm SEM from 3 independent experiments) show the percent of cells containing greater than 10 foci. The upper panel of $\mathbf{B}$ shows representative Rad51 foci immunofluorescence staining in irradiated and unirradiated mouse primary neurons. Original magnification, $\times 400$. ${ }^{\star} P<0.05$ versus control.

mum 17 fold; Figure 8B), which suggests that the intrinsic resistance to IR-induced DSBs in glioma cells may be, at least in part, caused by an enhanced capacity of glioma cells for DNA-PK-dependent repair. Rad51 foci were persistent and continued to increase in GL261 cells relative to hippocampal neuronal cells, particularly at 4 hours after IR (Figure 8C). Furthermore, IR-induced Rad51 foci formation was not affected by lithium pretreatment in these tumor cells, but was slightly suppressed in normal neuron cells. It would be interesting to determine whether glioma tumor cells also possess an enhanced capacity for HR in future studies. As HR is considered to be responsible for the repair of persistent complex lesions that cannot be resolved by simple NHEJ repair, glioma tumor cells may be capable of efficiently using both DNA repair systems to ensure survival after DNA insults. Furthermore, one could potentially take advantage of the differential induction of HR between normal and cancer cells to improve therapeutic index.

Despite its high resistance to therapy, the standard of care for gliomas still remains concurrent chemotherapy and cranial IR. In addition, CNS radiation continues to be a vital treatment modality for pediatric brain tumors and leukemia. Long-term morbidity from this treatment is devastating for these patients and their families and is of significant concern for the physician. Substantial evidence supports the idea of lithium-mediated neuroprotection from IR-induced neuronal apoptosis. Early results from our phase I trial suggest the feasibility of lithium treatment before and during cranial IR in patients with brain metastases (our unpublished observations). In addition, based on our findings in the present study, molecular-targeted therapies to enhance DNA repair may provide neuroprotection during cranial IR.

\section{Methods}

Cell culture. The mouse hippocampal neuronal cell line HT-22 was obtained from D. Schubert (Salk Institute, La Jolla, California, USA) and maintained in DMEM with $10 \% \mathrm{FBS}$ and $1 \%$ penicillin/streptomycin (Life Technologies). The human glioma cell line D54 and mouse glioma cell line GL261 was generously provided by Y. Gillespie (University of Alabama-Birmingham, Birmingham, Alabama, USA) and maintained in DMEM with Nutrient Mixture F-12 1:1, 10\% FBS, 1\% sodium pyruvate, and 1\% penicillin/streptomycin (Life Technologies). Cells were grown in a $5 \% \mathrm{CO}_{2}$ incubator at $37^{\circ} \mathrm{C}$.

Animal care and mouse neuronal tissue. All animal procedures were approved by the Vanderbilt University Institutional Animal Care and Use Committee. Mice were housed up to 5 per cage on a 12-hour light/12-hour dark cycle. Food (Purina Rodent Chow) and water were provided ad libitum. Timed pregnant C57BL/6J mice and 3-week-old SCID male mice (B6.CB17-Prkdc. scid/SzJ) were obtained from the The Jackson Laboratory. Lithium chloride was dissolved in PBS, and a 40-mg/kg dose was administered to mice via i.p. injection beginning on P7. Mice were treated with 3 Gy IR on P14. Prior to IR, 
A

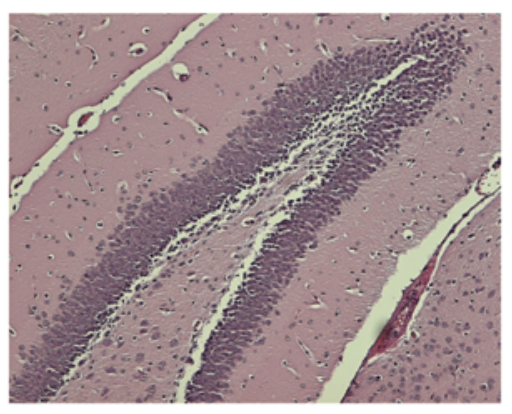

C

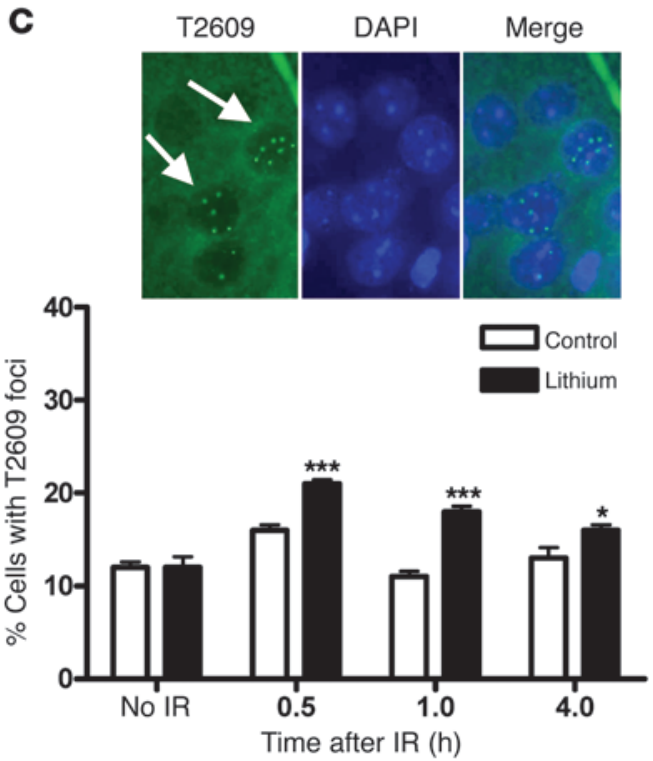

B
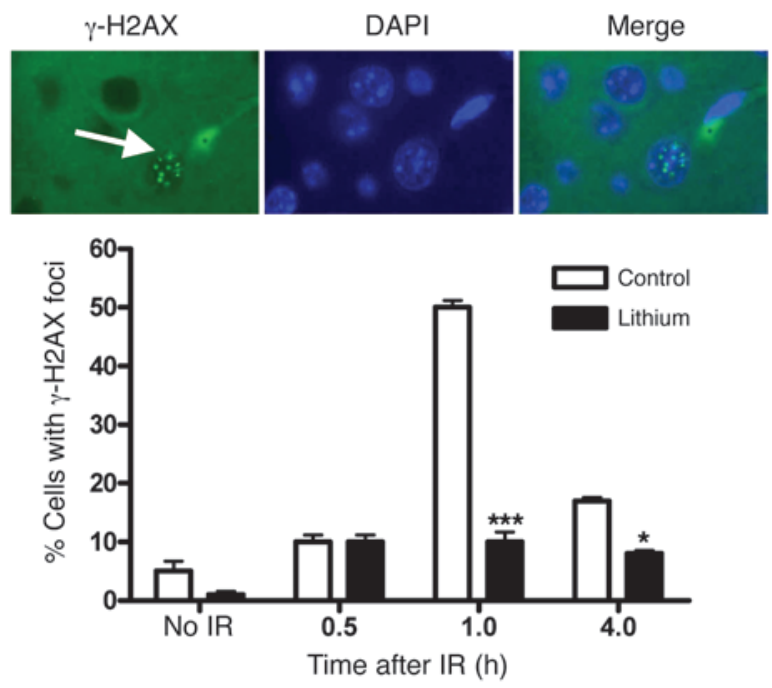

D
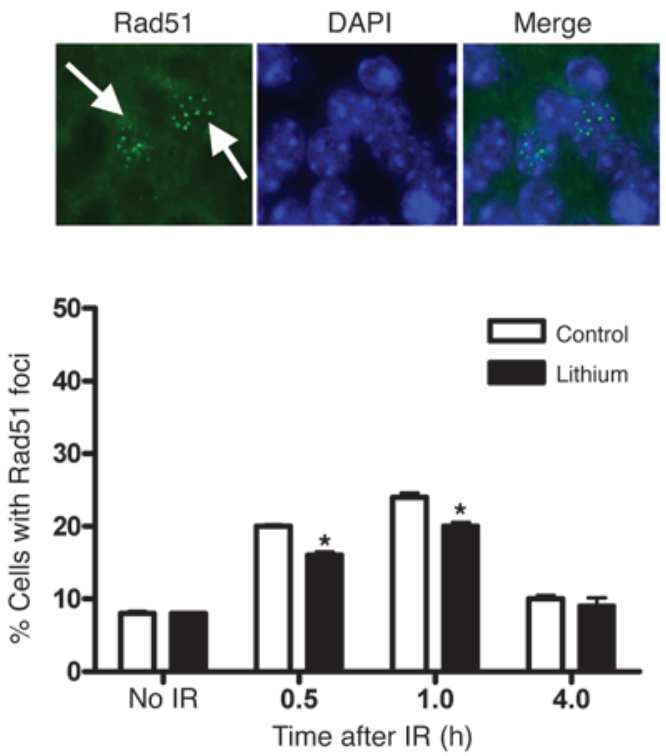

\section{Figure 6}

Lithium-mediated effects in C57BL/6J mice. (A) Representative H\&E-stained hippocampal tissue section. Original magnification, $\times 100$. (B-D) Foci immunofluorescence in irradiated mice with or without lithium prophylaxis. Mice were given $40 \mathrm{mg} / \mathrm{kg}$ lithium via i.p. injection beginning on P7 for 7 days. Following the prophylactic treatment period, mice were exposed to 3 Gy. At the indicated times, mouse hippocampal tissue was processed for immunofluorescence staining for (B) $\gamma$-H2AX, indicative of DSBs, (C) DNA-PK T2609, indicative of active NHEJ repair, or (D) Rad51, indicative of active HR repair. Data (mean \pm SEM) show the percentage of cells containing greater than 10 foci. For each foci analysis, 5 sections per mouse from a total of 5 mice were used. The upper panels show representative corresponding foci immunofluorescence staining in irradiated mice. Arrows indicate cells with foci. Original magnification, $\times 400 .{ }^{\star} P<0.05,{ }^{* \star} P<0.001$ versus control.

mice were anesthetized restrained in plastic tubing. Animals were exposed to cranial IR using a Therapax DXT $300 \mathrm{X}$-ray machine (Pantak Inc.) delivering $2.04 \mathrm{~Gy} / \mathrm{min}$ at $80 \mathrm{kVp}$. Mice were subsequently sacrificed 0.5 hours, 1 hour, and 4 hours after IR for DNA repair studies, or 6 hours and 12 hours after IR for apoptosis studies, by cervical dislocation under isoflurane anesthesia.

The brain was removed and placed in 10\% paraformaldehyde solution for 24 hours. The tissue was subsequently dehydrated. The frontal lobes were removed before embedding in paraffin. Sections were cut by microtome until the anterior hippocampus was visualized. Hippocampal tissue was verified by a neuropathologist. Coronal sections $(5 \mu \mathrm{m})$ were then taken and placed on Superfrost Gold Plus slides (Erie Scientific). Sections were then assessed via immunofluorescence for DNA repair $(\gamma$-H2AX, Rad51, and T2609 foci formation) or for apoptosis (cleaved caspase-3 and DAPI) as described below.

Primary culture of mouse neurons. Primary mouse neurons were isolated essentially as previously described (57). Briefly, E19-E21 fetuses were removed from pregnant C57BL/ 6 mice under deep anesthesia. Brains were dissected, and the hippocampus was isolated and placed in cold HBSS (Gibco, Invitrogen). Sections were cut into small pieces and placed in a protease-papain-DNase solu- 
A
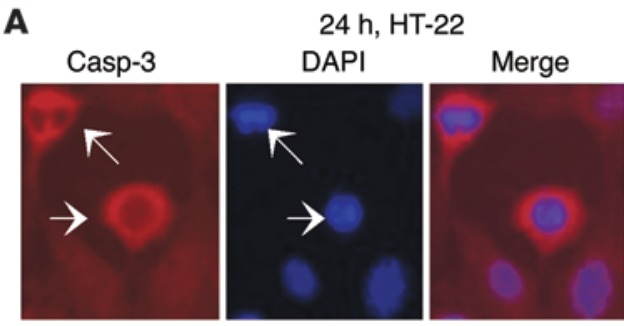

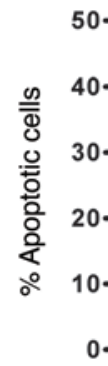

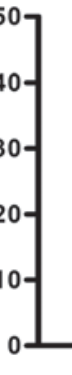

C

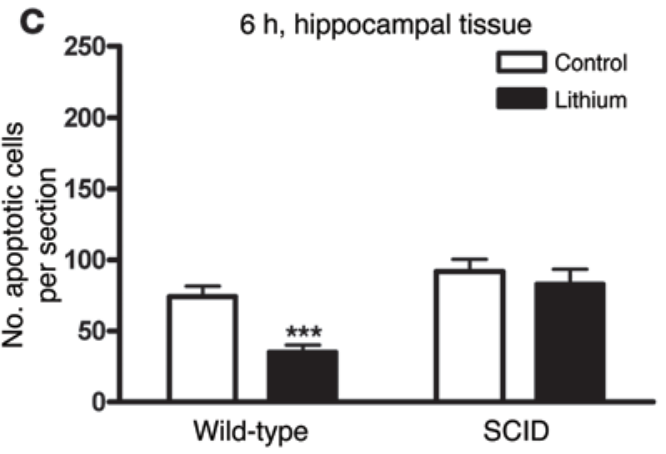

B

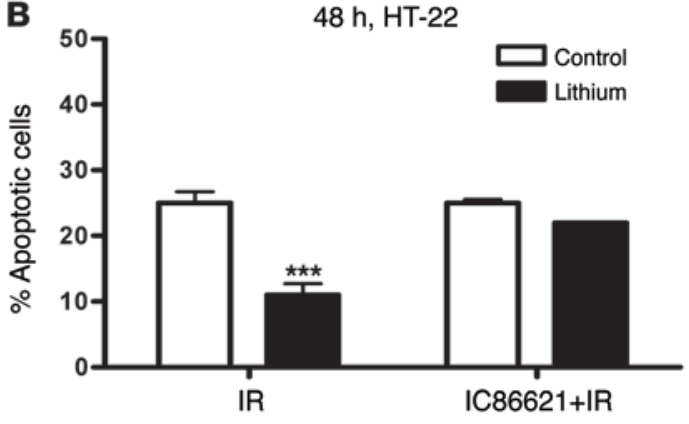

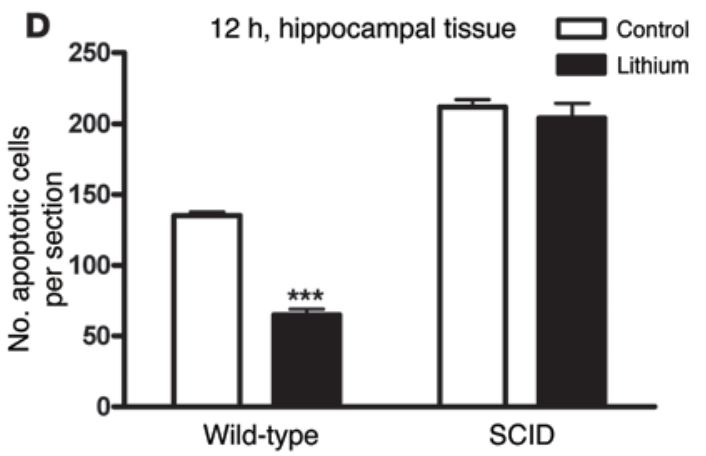

Figure 7

Lithium-mediated neuroprotection is dependent on DNA-PK. (A and B) Neuroprotection was attenuated by the DNA-PK inhibitor IC86621 in HT-22 mouse hippocampal cells. Cells were treated with $3 \mathrm{mM}$ lithium for 7 days. At 24 hours before IR, cells were exposed to the DNA-PK inhibitor IC86621 for 24 hours and subsequently irradiated with $3 \mathrm{~Gy}$. At the indicated times after IR, cells were processed for immunofluorescence staining with DAPI and cleaved caspase- 3 , indicative of apoptosis activation. Data (mean \pm SEM from 3 independent experiments) show percentage of cells with positive cleaved caspase-3 staining and pyknotic or condensed nuclei after subtracting respective basal apoptotic levels from each condition. The inset in A shows representative cleaved caspase-3 and DAPI staining in irradiated HT-22 hippocampal neurons. Arrows indicate cells with nuclear morphology, consistent with activation of the apoptotic pathway. Original magnification, $\times 400$. (C and D) Neuroprotection was abrogated in NHEJ repair-deficient SCID mice. Wild-type C57BL/6J or SCID mice were given $40 \mathrm{mg} / \mathrm{kg}$ lithium i.p. beginning at P7 for 7 days. Following the treatment period, mice were exposed to $3 \mathrm{~Gy}$. At the indicated times after IR, mouse hippocampal tissue was processed for immunofluorescence staining for cleaved caspase-3 and DAPI. Data (mean \pm SEM) show the total number of cells per section with positive staining for cleaved caspase-3. For each analysis, 3 sections per mouse from a total of 3 mice were used. ${ }^{* * *} P<0.001$ versus control.

tion for 30 minutes at $37^{\circ} \mathrm{C}$. The tissue was then gently triturated with a Pasteur pipet 10 times to dissociate neurons. Single cells were collected by $1,500 \mathrm{~g}$ centrifugation for 5 minutes. Cells were resuspended in a chemically defined medium (plating medium) consisting of DMEM, pyruvic acid, 20\% glucose, $200 \mathrm{mM}$ glutamine, 1:100 penicillin, 1:100 streptomycin, and 10\% FBS. Cell count was performed using a hemacytometer, and 15,000 cells $/ \mathrm{cm}^{2}$ were plated and maintained in neuronal medium (plating medium with $20 \mathrm{mM}$ glutamine). To confirm the neural origin of these cells, immunofluorescence was performed using a polyclonal antibody (catalog no. 10009506; Cayman Chemical) against postsynaptic density protein of $95 \mathrm{kDa}$ (PSD-95), a very prominent component of the postsynaptic densities of synapses (data not shown). Details of the immunofluorescence protocol are described below.

Immunohistochemistry. For DNA repair experiments, cells were cultured and mounted onto sterile glass slides. They were then treated with vehicle or $3 \mathrm{mM}$ lithium for 7 days. Following the treatment period, cells were exposed to either mock IR or 3 Gy IR using a Therapax DXT 300 X-ray machine (Pantak Inc.) delivering $2.04 \mathrm{~Gy} / \mathrm{min}$ at $80 \mathrm{kVp}$. At various time points after IP, immunohistochemistry for $\gamma$-H2AX, Rad51, and DNA-PK T2609 was performed as previously described (58). Primary antibodies include mouse anti-phospho- $\gamma$-H2AX antibody (diluted 1:1,000, catalog no. 07-164; Upstate), rabbit anti-Rad51 antibody (diluted 1:500, catalog no. PC130; Calbiochem), and mouse anti-T2609 antibody (diluted 1:100, catalog no. GTX18356; Genetex). Secondary antibodies include anti-mouse Alexa Fluor 488-conjugated antibody (diluted 1:1,000, catalog no. A-11059; Invitrogen), or anti-rabbit Alexa Fluor 488-conjugated antibody (diluted 1:1,000, catalog no. A-11008; Invitrogen). Total cells were counted under a fluorescent microscope ( $\times 400$ objective; Carl Zeiss), and cells containing greater than 10 foci were scored as positive. At least 500 cells were counted.

Hippocampal tissue sections were prepared as described above and immunostained using conditions similar to those for cultured cells described above. The total number of cells was counted under a light microscope ( $\times 400$ objective), and cells containing greater than 10 foci were mea- 

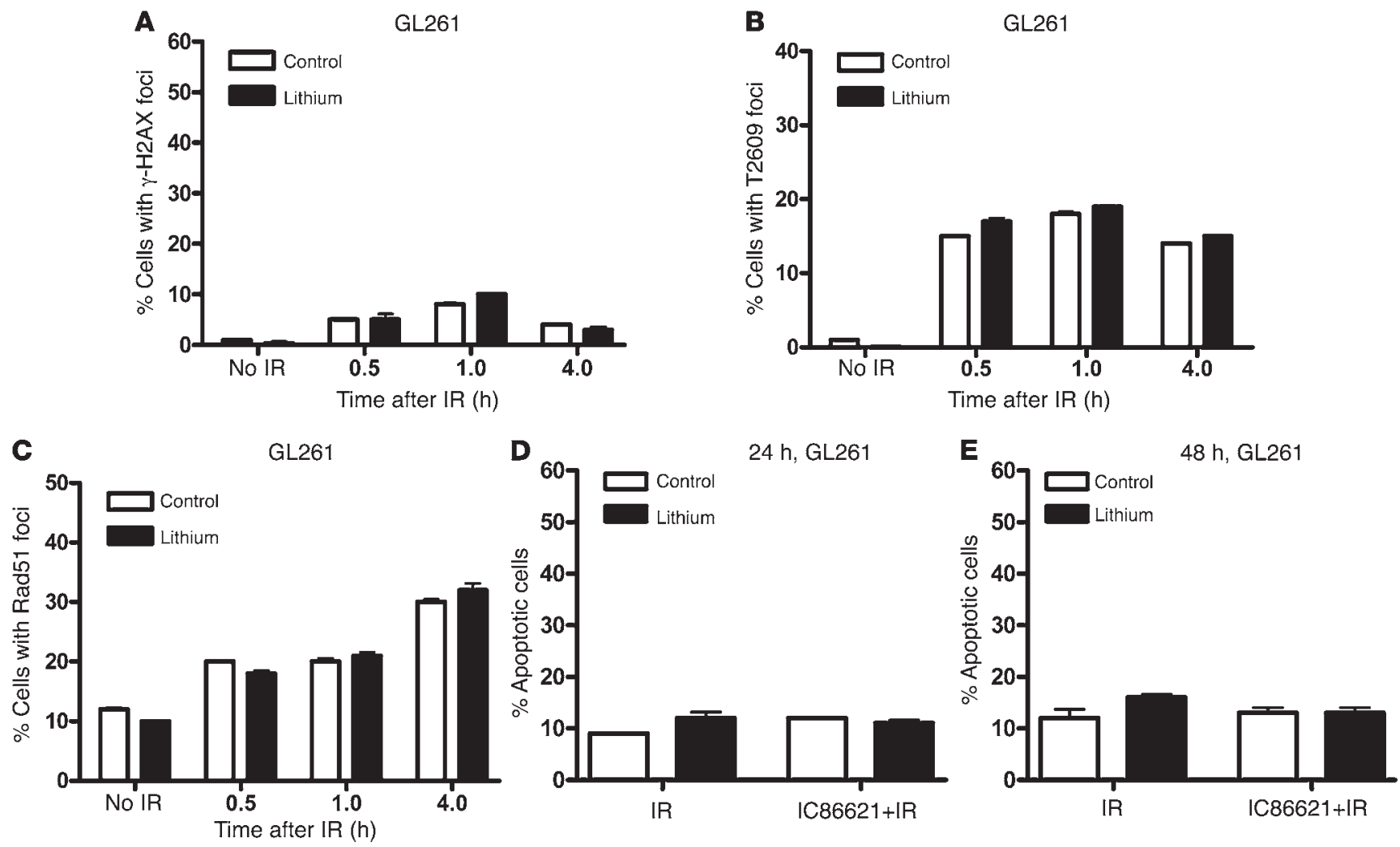

Figure 8

Lithium-mediated effects are not apparent in glioma tumor cells. Cells of the glioma cancer cell line GL261 were treated with $3 \mathrm{mM}$ lithium for 7 days. Following the treatment period, cells were exposed to 3 Gy. At the indicated times after IR, cells were processed for immunofluorescence staining for $\gamma-\mathrm{H} 2 \mathrm{AX}(\mathbf{A})$, DNA-PK T2609 (B), or Rad51 (C). Data (mean \pm SEM from 3 independent experiments) show the percentage of cells containing greater than 10 foci. Experiments were performed simultaneously with HT-22 cells $(\gamma-\mathrm{H} 2 \mathrm{AX}$, Figure 2A; T2609, Figure 3A; Rad51, Figure $5 A$ ). ( $\mathbf{D}$ and $\mathbf{E})$ Neuroprotection by lithium is not evident in glioma tumor cells and is unaffected by the addition of the DNA-PK inhibitor IC86621. Cells of the glioma cancer cell line GL261 were treated with $3 \mathrm{mM}$ lithium for 7 days. At 24 hours before IR, cells were exposed to the DNA-PK inhibitor IC86621 for 24 hours and subsequently irradiated with 3 Gy. At the indicated times after IR, cells were processed for immunofluorescence staining with DAPI and cleaved caspase- 3 , indicative of apoptosis activation. Data (mean \pm SEM from 3 independent experiments) show percentage of cells with positive cleaved caspase-3 staining and pyknotic or condensed nuclei after subtracting respective basal apoptotic levels from each condition.

sured using fluorescence microscopy (Carl Zeiss). At least 5 fields containing 50-100 cells per field were counted per section. A total of 5 sections per mouse from 5 mice per condition were used for each respective foci study.

For apoptosis experiments, HT-22 cells were also exposed to the DNA-PK inhibitor IC86621 (200 $\mu \mathrm{M}$; ICOS Corp.) or DMSO vehicle control for 24 hours prior to IR. Cells were then costained with DAPI $(1 \mu \mathrm{g} / \mathrm{ml})$ and cleaved caspase-3 (rabbit anti-caspase-3, diluted 1:250, catalog no. 9661; Cell Signaling) and Cy3-conjugated anti-rabbit IgG (diluted 1:200, catalog no. C-2306; Sigma-Aldrich). Staining patterns were visualized by fluorescence microscopy (Carl Zeiss). Cells with condensed nuclei and cleaved caspase-3-positive staining were scored as apoptotic cells. Positive and negative controls were included on all experiments. At least 500 cells were assessed.

Hippocampal tissue sections from both wild-type C57BL/6J and SCID mice were prepared as described above. The sections were stained with $\mathrm{H} \& \mathrm{E}$ in the standard fashion. Cleaved caspase- 3 and DAPI costaining was performed as described above. The superior curvature of the hippocampus (Figure 6A) was used for quantification of apoptosis in all cases. The subgranular zone was identified as a layer, $2-3$ cells thick, adjacent to the granular cell layer facing the hilus. Apoptotic cells were counted in these tissue sections prepared throughout the hippocampi of mice at various cutting depths. The mean number of total apoptotic cells per hippocampal section was obtained from 3 tissue sections per mouse and 3 mice per treatment group.

Nentral comet assay. HT-22 neurons were treated with $3 \mathrm{mM}$ lithium or vehicle for 7 days. Following the treatment period, cells were exposed to 3 Gy and incubated for various times, after which they were prepared and subjected to neutral CometAssay according to the manufacturer's instructions (catalog no. 4250-050-K; Trevigen). Briefly, cells were combined with low melting agarose onto CometSlides (Trevigen). After lysis, cells were subjected to electrophoresis. Cells were then visualized using fluorescent microscopy (Carl Zeiss). At least 75 comet images were obtained for each time point and analyzed using Comet Score software (version 1.5; TriTek Corp.). Experiments were repeated in triplicate. Data show the percentage of cells with comet tail as well as mean comet tail moment and SEM.

Assay of NHEJ repair by circularization of linear plasmid substrate. A single DSB was generated in the plasmid substrate pEGFP-N1 (Clontech) by cleavage between the promoter and GFP reporter gene with EcoRI (Figure 4A). Linearized DNA was gel purified for cell transfection. Cells were cotransfected with pdsRed (Clontech), as a transfection efficiency internal control, and with cleaved substrate using Lipofectamine2000 (Roche). Cells were harvested 48 hours later and subjected to 2 -color fluorescence analysis. The green fluo- 
rescent cells represented the repaired DSB and restoration of GFP expression. The red fluorescent cells represented exogenous DNA transfection efficiency. For each analysis, 100,000 cells were processed. The relative DSB rejoining activity was obtained by the ratio of green to red fluorescent cells.

Statistics. Data were analyzed via 2-way ANOVA followed by a Bonferroni post-test using Prism for Windows (version 4.02; GraphPad Software). A $P$ value less than 0.05 was considered significant.

\section{Acknowledgments}

The authors thank Dinesh Thotala for assistance with hippocampal tissue sections. We also appreciate the help of Juhong Jiang with animal work. This work was supported by grant RR0725 from the Radiological Society of North America (to E.S. Yang).

Received for publication September 25, 2007, and accepted in revised form February 11, 2009.

Address correspondence to: Fen Xia, Department of Radiation Oncology, Vanderbilt University Medical Center, The Vanderbilt Clinic, 1301 22nd Avenue South, B-902 TVC, Nashville, Tennessee 37232-5671, USA. Phone: (615) 322-2555; Fax: (615) 343-0161; E-mail: fen.xia@vanderbilt.edu.
1. DeAngelis, L.M., Delattre, J.Y., and Posner, J.B. 1989. Radiation-induced dementia in patients cured of brain metastases. Neurology. 39:789-796

2. Surma-aho, O., et al. 2001. Adverse long-term effects of brain radiotherapy in adult low-grade glioma patients. Neurology. 56:1285-1290.

3. Kiehna, E.N., Mulhern, R.K., Li, C., Xiong, X., and Merchant, T.E. 2006. Changes in attentional performance of children and young adults with localized primary brain tumors after conformal radiation therapy. J. Clin. Oncol. 24:5283-5290.

4. Bowers, D.C., et al. 2006. Late-occurring stroke among long-term survivors of childhood leukemia and brain tumors: a report from the Childhood Cancer Survivor Study. J. Clin. Oncol. 24:5277-5282.

5. Deweer, B., Pillon, B., Pochon, J.B., and Dubois, B. 2001. Is the HM story only a "remote memory"? Some facts about hippocampus and memory in humans. Behav. Brain Res. 127:209-224.

6. Yazlovitskaya, E.M., et al. 2006. Lithium treatment prevents neurocognitive deficit resulting from cranial irradiation. Cancer Res. 66:11179-11186.

7. Wood, G.E., Young, L.T., Reagan, L.P., Chen, B., and McEwen, B.S. 2004. Stress-induced structural remodeling in hippocampus: prevention by lithium treatment. Proc. Natl. Acad. Sci. U. S. A. 101:3973-3978.

8. Abayomi, O.K. 2002. Pathogenesis of cognitive decline following therapeutic irradiation for head and neck tumors. Acta Oncol. 41:346-351.

9. Rich, T., Allen, R.L., and Wyllie, A.H. 2000. Defying death after DNA damage. Nature. 407:777-783.

10. Olive, P.L. 1999. DNA damage and repair in individual cells: applications of the comet assay in radiobiology. Int. J. Radiat. Biol. 75:395-405.

11. Olive, P.L., and Banath, J.P. 2006. The comet assay: a method to measure DNA damage in individual cells. Nat. Protoc. 1:23-29.

12. Khanna, K.K., and Jackson, S.P. 2001. DNA doublestrand breaks: signaling, repair and the cancer connection. Nat. Genet. 27:247-254.

13. van Gent, D.C., Hoeijmakers, J.H., and Kanaar, R. 2001. Chromosomal stability and the DNA double-stranded break connection. Nat. Rev. Genet. 2:196-206

14. Iliakis, G., et al. 2004. Mechanisms of DNA double strand break repair and chromosome aberration formation. Cytogenet. Genome Res. 104:14-20.

15. Lieber, M.R., Ma, Y., Pannicke, U., and Schwarz, K. 2003. Mechanism and regulation of human nonhomologous DNA end-joining. Nat. Rev. Mol. Cell Biol. 4:712-720.

16. Chan, D.W., et al. 2002. Autophosphorylation of the DNA-dependent protein kinase catalytic subunit is required for rejoining of DNA doublestrand breaks. Genes Dev. 16:2333-2338.

17. Frank, K.M., et al. 2000. DNA ligase IV deficiency in mice leads to defective neurogenesis and embryonic lethality via the p53 pathway. Mol. Cell. 5:993-1002.

18. Gao, Y., et al. 1998. A critical role for DNA end-joining proteins in both lymphogenesis and neurogenesis. Cell. 95:891-902.

19. Sekiguchi, J., et al. 2001. Genetic interactions between ATM and the nonhomologous end-joining factors in genomic stability and development. Proc. Natl. Acad. Sci. U. S. A. 98:3243-3248.

20. Sekiguchi, J.M., et al. 1999. Nonhomologous endjoining proteins are required for $\mathrm{V}(\mathrm{D}) \mathrm{J}$ recombination, normal growth, and neurogenesis. Cold Spring Harb. Symp. Quant. Biol. 64:169-181.

21. Manji, H.K., Moore, G.J., and Chen, G. 1999. Lithium at 50: have the neuroprotective effects of this unique cation been overlooked? Biol. Psychiatry. 46:929-940.

22. Manji, H.K., Moore, G.J., and Chen, G. 2000. Lithium up-regulates the cytoprotective protein $\mathrm{Bcl}-2$ in the CNS in vivo: a role for neurotrophic and neuroprotective effects in manic depressive illness. J. Clin. Psychiatry. 61(Suppl. 9):82-96.

23. Ren, M., Senatorov, V.V., Chen, R.W., and Chuang, D.M. 2003. Postinsult treatment with lithium reduces brain damage and facilitates neurological recovery in a rat ischemia/reperfusion model. Proc. Natl. Acad. Sci. U. S. A. 100:6210-6215.

24. Chen, R.W., and Chuang, D.M. 1999. Long term lithium treatment suppresses p53 and Bax expression but increases Bcl-2 expression. A prominent role in neuroprotection against excitotoxicity. J. Biol. Chem. 274:6039-6042.

25. Bauer, M., Alda, M., Priller, J., and Young, L.T. 2003. Implications of the neuroprotective effects of lithium for the treatment of bipolar and neurodegenerative disorders. Pharmacopsychiatry. 36(Suppl. 3):S250-S254.

26. Jope, R.S. 2003. Lithium and GSK-3: one inhibitor, two inhibitory actions, multiple outcomes. Trends Pharmacol. Sci. 24:441-443.

27. Klein, P.S., and Melton, D.A. 1996. A molecular mechanism for the effect of lithium on development. Proc. Natl. Acad. Sci. U. S. A. 93:8455-8459.

28. Olive, P.L. 1999. DNA damage and repair in individual cells: applications of the comet assay in radiobiology. Int. J. Radiat. Biol. 75:395-405.

29. Kashishian, A., et al. 2003. DNA-dependent protein kinase inhibitors as drug candidates for the treatment of cancer. Mol. Cancer Ther. 2:1257-1264.

30. Zhuang, J., et al. 2006. Checkpoint kinase 2-mediated phosphorylation of BRCA1 regulates the fidelity of nonhomologous end-joining. Cancer Res. 66:1401-1408

31. Tashiro, S., Walter, J., Shinohara, A., Kamada, N., and Cremer, T. 2000. Rad51 accumulation at sites of DNA damage and in postreplicative chromatin. J. Cell Biol. 150:283-291.

32. Allen, C., Kurimasa, A., Brenneman, M.A., Chen, D.J., and Nickoloff, J.A. 2002. DNA-dependent protein kinase suppresses double-strand break-induced and spontaneous homologous recombination. Proc. Natl. Acad. Sci. U. S. A. 99:3758-3763.

33. Cowell, I.G., Durkacz, B.W., and Tilby, M.J. 2005. Sensitization of breast carcinoma cells to ionizing radiation by small molecule inhibitors of DNAdependent protein kinase and ataxia telangiectasia mutated. Biochem. Pharmacol. 71:13-20.

34. Daido, S., et al. 2005. Inhibition of the DNA-dependent protein kinase catalytic subunit radiosensitiz- es malignant glioma cells by inducing autophagy. Cancer Res. 65:4368-4375.

35. Shinohara, E.T., et al. 2005. DNA-dependent protein kinase is a molecular target for the development of noncytotoxic radiation-sensitizing drugs. Cancer Res. 65:4987-4992.

36. Kirchgessner, C.U., et al. 1995. DNA-dependent kinase (p350) as a candidate gene for the murine SCID defect. Science. 267:1178-1183.

37. Szatmári, T., et al. 2006. Detailed characterization of the mouse glioma 261 tumor model for experimental glioblastoma therapy. Cancer Sci. 97:546-553.

38. Banath, J.P., Macphail, S.H., and Olive, P.L. 2004. Radiation sensitivity, H2AX phosphorylation, and kinetics of repair of DNA strand breaks in irradiated cervical cancer cell lines. Cancer Res. 64:7144-7149.

39. Thotala, D.K., Hallahan, D.E., and Yazlovitskaya, E.M. 2008. Inhibition of glycogen synthase kinase 3 beta attenuates neurocognitive dysfunction resulting from cranial irradiation. Cancer Res. 68:5859-5868.

40. Mayo, L.D., and Donner, D.B. 2001. A phosphatidylinositol 3-kinase/Akt pathway promotes translocation of $\mathrm{Mdm} 2$ from the cytoplasm to the nucleus. Proc. Natl. Acad. Sci. U. S. A. 98:11598-11603.

41. Tran, H., et al. 2002. DNA repair pathway stimulated by the forkhead transcription factor FOXO3a through the Gadd45 protein. Science. 296:530-534.

42. Bozulic, L., Surucu, B., Hynx, D., and Hemmings, B.A. 2008. PKBalpha/Akt1 acts downstream of DNA-PK in the DNA double-strand break response and promotes survival. Mol. Cell. 30:203-213.

43. Tan, J., and Hallahan, D.E. 2003. Growth factorindependent activation of protein kinase B contributes to the inherent resistance of vascular endothelium to radiation-induced apoptotic response. Cancer Res. 63:7663-7667.

44. Feng, J., Park, J., Cron, P., Hess, D., and Hemmings, B.A. 2004. Identification of a PKB/Akt hydrophobic motif Ser-473 kinase as DNA-dependent protein kinase. J. Biol. Chem. 279:41189-41196.

45. Surucu, B., Bozulic, L., Hynx, D., Parcellier, A., and Hemmings, B.A. 2008. In vivo analysis of protein kinase B (PKB)/Akt regulation in DNAPKcs-null mice reveals a role for PKB/Akt in DNA damage response and tumorigenesis. J. Biol. Chem. 283:30025-30033.

46. Lu, D., Huang, J., and Basu, A. 2006. Protein kinase Cepsilon activates protein kinase B/Akt via DNAPK to protect against tumor necrosis factor-alphainduced cell death. J. Biol. Chem. 281:22799-22807.

47. Rossig, L., et al. 2001. Akt-dependent phosphorylation of p21(Cip1) regulates PCNA binding and proliferation of endothelial cells. Mol. Cell. Biol. 21:5644-5657.

48. Rossig, L., Badorff, C., Holzmann, Y., Zeiher, A.M., and Dimmeler, S. 2002. Glycogen synthase kinase-3 couples AKT-dependent signaling to the regulation of p21Cip1 degradation. J. Biol. Chem. 277:9684-9689.

49. Li, Y., Dowbenko, D., and Lasky, L.A. 2002. AKT/ PKB phosphorylation of p21Cip/WAF1 enhances protein stability of $\mathrm{p} 21 \mathrm{Cip} / \mathrm{WAF} 1$ and promotes 
cell survival. J. Biol. Chem. 277:11352-11361.

50. Boehme, K.A., Kulikov, R., and Blattner, C. 2008. p53 stabilization in response to DNA damage requires Akt/PKB and DNA-PK. Proc. Natl. Acad. Sci. U. S. A. 105:7785-7790.

51. Wang, X.M., et al. 2008. Involvement of the role of Chk1 in lithium-induced G2/M phase cell cycle arrest in hepatocellular carcinoma cells. J. Cell. Biochem. 104:1181-1191.

52. Wang, J.S., et al. 2008. Lithium inhibits proliferation of human esophageal cancer cell line Eca-109 by inducing a G2/M cell cycle arrest. World J. Gastroenterol. 14:3982-3989.
53. Pizarro, J.G., et al. 2008. A molecular study of pathways involved in the inhibition of cell proliferation in neuroblastoma $\mathrm{B} 65$ cells by the GSK-3 inhibitors lithium and SB-415286. J. Cell. Mol. Med. Online publication ahead of print. doi:10.1111/j.15824934.2008.00389.x.

54. Chuang, D.M., et al. 2002. Neuroprotective effects of lithium in cultured cells and animal models of diseases. Bipolar Disord. 4:129-136.

55. Marini, J.L., and Sheard, M.H. 1976. Sustainedrelease lithium carbonate in double-blind study: serum lithium levels, side effects, and placebo response. J. Clin. Pharmacol. 16:276-283.
56. Keck, P.E., Jr., and McElroy, S.L. 2002. Clinical pharmacodynamics and pharmacokinetics of antimanic and mood-stabilizing medications. J. Clin. Psychiatry. 63(Suppl. 4):3-11.

57. Zhou, F.C., Kelley, M.R., Chiang, Y.H., and Young, P. 2000. Three to four-year-old nonpassaged EGF-responsive neural progenitor cells: proliferation, apoptosis, and DNA repair. Exp. Neurol. 164:200-208.

58. Feng, Z., Kachnic, L., Zhang, J., Powell, S.N., and Xia, F. 2004. DNA damage induces p53dependent BRCA1 nuclear export. J. Biol. Chem. 279:28574-28584 